\title{
Surgery in Perinatology
}

\author{
E M Halimun
}

(Harapan Kita Children \& Maternity Hospital, Jakarta)

ABsTRACT Although modern neonatal surgery is a very young subject, it started only after the second world war, the progress is remarkable. The well intergrated interdisciplinary team among pediatric surgeons, pediatricians, neonatologits, anaesthetists, and other relevant specialists made this progress possible. Neonatal surgey has been influenced by the advances in prenatal diagnosis. Neonatal surgical emergencies are related to perinatal conditions such as better antenatal care, including ultrasonogram examination, encourage the high risk pregnant women to deliver at pediatric/perinatal centers where obstetricians, pediatricians or neonatologist, pediatric surgeon, anesthetist are working as a team. Prenatal diagnosis has been one of the most challenging aspects in perinatology, and may directly related to the task of the pediatric surgeons to salvage certain type of malformations. Some experience of perinatal surgery is also discussed. [Paediatr Indones 1995; 35:205-210]

\section{Introduction}

It has been generally recognized that surgery in perinatology is the very essence of pediatric surgery. This has never been disputed between pediatric surgeons on one side and general surgeons or organ specialists (urologist, plastic surgeon, etc.) on the other. It has always been agreed that neonatal surgery belongs to the pediatric surgeons.

"Presented at The Panel Discussion of the International Conference on Recent Advances in Perinatology, Yogyakarta-Indonesia, July 1992.
Although modern neonatal surgery is a very young subject, it started only after the second world war, the progress is remarkable. The well integrated interdisciplinary team among pediatric surgeons, pediatricians, neonatologists, anesthetists, and other relevant specialists made this progress possible.

In this brief review I shall discuss two subjects which are of utmost important, i.e.,

- advances in surgical intervention in perinatology, and

- present status of neonatal surgery in Indonesia 


\section{Advances in Surgical Intervention in Perinatology}

The advent of prenatal diagnosis positively alters the attitude toward the tim ing of surgical intervention in perinatol ogy. The modern methods of prenatal diagnosis, especially ultrasonography, amniocenteses and fetoscopy, provide us with early information concerning the clinical condition of the fetus. Early recognition of congenital malformation in utero may be the basis for many important decisions regarding the future of the mother and child. Some fetal malformations with a known pattern of inheritance may be specifically sought for. However some obstetric conditions such as oligohydramnios and polyhydramnios are associated with underlying fetal malformations. In the past, the only question raised by the prenatal diagnosis of a fetal malformation was whether to abort the fetus or to wait delivery. Nowadays other therapeutic alternatives are available such as changing the mode of delivery and even intrauterine treatment.

The rationale for determining how the prenatal diagnosis of any given fetal defect will affect perinatal management is as follows:

1. incompatible with life: abort

2. dystocia: cesarean section (CS)

3. progressive ill effect: delivery early

4. correctable at term: wait

5. alter development: correction in utero

Lesions incompatible with postnatal life suggesting mother abortion. In most surgically correctable cases transport to a pe rinatal center where neonatologists, ped atric anesthetists, and neonatal surgeons are available is recommended. Le- sions causing disproportion between fe tus and the mother's pelvis require cesar ean delivery.

A few lesions that cause progressive harm to a certain organ may require ear ly delivery for immediate repair outside the uterus. Lesions causing progressive disturbance on the organ development of the fetus may require intrauterine treat ment.

Malformation usually managed by selective abortion (pregnancy

\section{termination)}

When serious organ defects incompati ble with normal postnatal life are diag nosed early enough, the family has the option of terminating the pregnancy. When the malformations are detected too late for safe abortion, the family should be counseled and appropriate and agreeable postnatal management arranged. The examples of the severe defect that may be considered indications for selective termination are listed in Table 1.

Some of these chromosomal defects can be diagnosed by culture of amniotic fluid cells. Some anatomic defects and developmental abnormalities can be diagnosed by regular prenatal sonography.

\section{Defects that may influence the mode of delivery}

Elective cesarean delivery may be indicated in fetal malformations listed in Table 2 . These malformations mostly cause dystocia. Other indication for selective CS is a malformation requiring immediate surgical correction in a sterile condition, such as in uncovered meningomyelocele.
$\mathrm{CS}$ is also indicated in cases of malformation with fetal distress.

Table 1. Malformations usually managed by selective abortion

- Anencephaly, porencephaly and giant hydrocephalus

- Multiple anomalies associated with chromosomal abnormalities (e.g. trisomy 13 or 18)

- Bilateral agenesis or polycystic kidney disease

Uncorrectable inherited metabolic or chromosomal disorders (hemoglobinopathies, Tay-Sachs disease etc.)

Table 2. Malformations that may require $\mathrm{Ce}$ -

sarean delivery

- Conjoined twins

- Giant omphalocele, gastroschizis

- Large hydrocephalus

- Large sacrococcygeal teratoma

- Large cystic hygroma

- Malformation with fetal distress

\section{Defect that can influence the timing of delivery}

Early delivery may be indicated for certain fetal malformations that require correction as soon as possible after antenata diagnosis. In this condition the risk of premature delivery must be considered against the risk of continuing the preg nancy. In some malformations the pro- gressive ill effects to the fetus start directly in utero. In case of amniotic band complex, a part of the fetus is compressed or strangulated by herniation through defect in the fet 1 membrane resulting in deformity or ainputation. This simple mechanical restriction to growth must immediately be relieved to prevent deformity. Nowadays, the outcome of premature infant with RDS could be relieved by the stimulating fetal surfactant production with corticosteroids in ventilator.

Table 3. Anomalies that may require induced preterm delivery for early correction

- Obstructive hydronephrosis

- Obstructive hydrocephalus

- Gastroschizis

- Strangulated intestinal obstruction (volvulus)

\section{Malformation best corrected after at} term delivery

Ideally, malformations that can be diagnosed in utero are best managed by appropriate surgical and medical therapy after at term delivery. The term infants have a better anesthetic and surgical risk than the preterm infants. In correctable malformation detected in utero, the delivery can be planned so that appropriate facilities (anesthesiologist, neonatologist, pediatric surgeon) are available. Delivery in a perinatology center is recommended; transporting the fetus in situ (maternal transport) is preferable to postnatal transport of the high risk newborn. 
Table 4. Malformations best corrected after term delivery

\section{- G I tract atresia}

- Small omphalocele

- Small meningocele

- Unilateral hydronephrosis

- Small hygroma or coccygeal teratoma

- Benign cysts (ovarial, choledochal cyst etc.)

\section{Malformation that may require intra-} uterine intervention

The anatomic malformations that warrant consideration are those that interfere the fetal organ development and if released would allow normal fetal development to proceed. Malformation that deserve consideration for fetal surgery are listed in Table 5.

Table 5. Anatomic defects that interfere with development and may require fetal surgery

Malformation Effect on development

- Urinary tract hydronephrosis obstruction renal and resp. failure

- Lung hypoplasia resp failure

- Cerebrospinal brain damage

fluid obstruction hydrocephalus

- Diaphragmatic lung hypoplasia hernia respiratory failure

(from unborn patient: MR Harrison et al 1984)

In conclusion: Recent advances in prenatal diagnosis offers new hope for im- proving the management of the fetus with correctable congenital malformation However, the more invasive diagnostic and therapeutic procedures may cause significant risks for both fetus and mother, raising difficult ethical questions about risks versus benefits and about the right of the fetus and mother.

\section{Neonatal Surgery in Indonesia}

In Indonesia up till 1992 there were only 15 pediatric surgeons serving a country with the population (census on 1990) of $180,000,000$ population with the birth rate of 30 per 1000 mothers; it means that there will be 5,1 million newborn ba bies per year. Unfortunately the infant death rate (1990) is still high, namely 50 per 1000 live births.

Neonatal surgical cases may be group ed into:

1. Congenital malformations

2. Neonatal surgical emergencies related to perinatal conditions

\section{Congenital malformations}

Inborn malformations which need immediate surgical intervention in the neonatal period are listed in Table 6.

\section{Abdominal wall defect}

Gastroschizis and omphalocele, even if treated in the pediatric surgical center still give a high mortality rate, i.e., up to $45 \%$. These cases usually present themselves to the surgeons with hypothermia, dryness and contaminated bowel/surface. These conditions are due to poor transformation and hygiene.

\section{Surgical respiratory distress syndrome}

Cases like lobar emphysema and cystic lung disease are not common finding in the pediatric surgical centers. It is not a really uncommon defect but mostly du to failure of the primary health personne to detect the disease.

Table 6. Congenital malformations requiring mediate surgery in neonatal period

- Abdominal wall defect: gastroschisis, omphalocele

- Surgical resiratory distress syndrome

e.g - lobar emphysema - cystic lungs

- pneumothorax

- diaphragmatic hernia

- Esophago-gastrointestinal obstruction atresia

- Urinary tract obstruction, e.g - uretro-pelvic junction stenosis - posterior valve

- Blue babies caused by cardiovascular defects

- Progressive CSF obatruction

Blue babies and CSF obstruction are beyond the competence of pediatric surgeons.

\section{Esophago-gastrointestinal atresias}

With the increasing alertness of the pediatricians and neonatologists in early diagnosis of the patency of gastrointestinal tract, nowadays we can see an increasing survival rate from $60 \%$ in 1970 's to $80-90 \%$ in 1991 .

\section{Urinary tract obstruction}

Unilateral or bilateral hydronephrosis is the result of urinary tract obstruction. Early surgical intervention in the neonatal period gives a good prognosis. Unfortunately in this country usually these cases ate neglected until the babies become older before going to surgery.

\section{Neonatal surgical emergencies} related to perinatal conditions

The development of prenatal diagnostic teams and neonatal intensive care services in pediatric centers in Indonesia have resulted in the survival of many seriously ill newborns, which might cause another serious complications in infancy such as necrotizing enterocolitis (NEC), pneumothorax, and secondary intracranial bleeding.

In 1991 at the Children and Maternity hospital Harapan Kita, Jakarta, we found 75 surgical cases among neonates treated in the level II and III wards:

a 47 cases of NEC

a 3 cases of spontaneous pneumothorax

- 25 secondary cerebral bleeding

Fourty seven cases or $96 \%$ of NEC cases were treated conservatively by parental nutrition for more than 7 days, nasogastric drainage and antibiotics with a survival rate of $55 \%$. Two cases or NEC underwent sungery with the indication of worsening after the conservative treatment, persistence of bowel loop or signs of pneumoperitoneum. Only one case survived. Spontaneous pneumothorax is a predicted complication after intensive respiratory care. Most of the pneumothorax cases resolved spontaneously. Only three cases needed thorax drainage, 
which gave a good relieve. Secondary cerebral bleeding needs a long term followup if the patients survive from the primary disease. Only cases with secondary progressive intracranial tension needs immediate VP shunting.

\section{Conclusions}

To improve the outcome of neonatal surgical cases in Indonesia, one should do:

- a better antenatal care, including ultrasonogram examination

- encourage the high risk pregnant women to deliver at pediatric/perinatal centers where obstetricians, pediatricians or neonatologists, pediatric surgeons, anesthetists are working as a team. Maternal transport is better than ill newborn transport

- educating the physician, midwives, nursing staff in the primary health centers in early diagnosis on high risk pregnancy and life threatening congenital malformation improve the quantity and quality of pediatric surgeons in Indonesia

\section{References}

1. Adzick NS, Flake AW, Harrison MR. Recent advance in prenatal diagnosis and treatment, Pediatr Clin North Am 1985: 32:1103.

2. Halimun EM, Ganung NP. Pediatric surgical services in rural population. Presented at the Symposium of Pediatric Surgery in Rural Country. Asian Association of Pediatric Surgeons. Tokyo March 12, 1992.

3. Harrison MR, Golbus MS, Filly RA. Management of the fetus with a correc- tible congenital defect. JAMA 1981; 246: 774.

4. Harrison MR, Golbus MS, Filly RA. Unborn patient; 2nd ed. Orlando, Fl. Grune Stratton 1989.

5. Holschneider AM, Baumgartner M, Mascott C. Consequences of antenatal diagnosis for pediatric surgery. Progr Pediatr Surg 19:184, 1986.

6. Rickham PP. Thoughts about the past and future of neonatal surgery. J Pediatr Surg 27:1,1992. 